\title{
Modifications of adiposity in school-age children according to nutritional status: a 20-year analysis
}

\author{
Modificações da adiposidade em escolares de acordo \\ com o estado nutricional: análise de 20 anos \\ Tatiane K. Ferrari ${ }^{1}$, Gerson Luis de M. Ferrari' ${ }^{1}$, João Pedro da Silva Júnior ${ }^{2}$,
Leonardo José da Silva ${ }^{3}$, Luís C. Oliveira ${ }^{3}$, Victor K. R. Matsudo ${ }^{4}$
}

\section{Resumo}

Objetivo: Analisar as modificações da adiposidade de escolares durante 20 anos, de acordo com o estado nutricional.

Métodos: $O$ estudo faz parte do Projeto Misto Longitudinal de Crescimento, Desenvolvimento e Aptidão Física de Ilhabela. Uma amostra de 1.095 escolares de ambos os sexos, de 7 a 10 anos, atendiam aos seguintes critérios de inclusão: (a) pelo menos uma avaliação completa em um dos períodos analisados; (b) estar no estágio pré-púbere de maturação sexual; (c) ser aparentemente saudável. Os períodos analisados foram 1990/1991 (inicial), 2000/2001 (10 anos) e 2010/2011 (20 anos). As variáveis analisadas foram: massa corporal, estatura e adiposidade, pela análise individual de cada dobra cutânea. Os indivíduos foram classificados em eutróficos, com excesso de peso e obesos mediante as curvas propostas pela Organização Mundial da Saúde de índice de massa corporal para idade e sexo. Foi utilizada a Anova one-way, seguida do post-hoc de Scheffé, com $\mathrm{p}<0,01$.

Resultados: Nos meninos, o maior aumento ocorreu no grupo com excesso de peso, seguido pelos grupos obesos e eutróficos. Já nas meninas, os maiores aumentos ocorreram nos grupos com excesso de peso e eutróficas, seguidos pelo grupo obesas.

Conclusão: Houve modificações da adiposidade, mesmo com o controle do estado nutricional, durante o período de 20 anos analisado, mostrando que os indivíduos que têm semelhantes índices de massa corporal podem variar em proporção e distribuição de tecido adiposo subcutâneo. Para ambos os sexos, o aumento foi maior nos grupos classificados como excesso de peso e, principalmente, nas dobras cutâneas centrais.

J Pediatr (Rio J). 2012;88(3):239-45: Pregas cutâneas, tecido adiposo, pré-escolar.

\begin{abstract}
Objective: To analyze adiposity changes in school-age children over a 20 year-period, according to nutritional status.

Methods: The study is part of the Ilhabela Longitudinal Mixed Project on Growth, Development and Physical Fitness. A sample of 1,095 school students of both sexes, from 7 to 10 years, met the following inclusion criteria: (a) at least one complete assessment in one of the analyzed periods; (b) to be in prepubertal stage of sexual maturation; (c) to be apparently healthy. The periods analyzed were 1990/1991 (initial), 2000/2001 (10 years) and 2010/2011 (20 years). The variables analyzed were: body weight, height and adiposity through individual analysis of each skinfold. Children were classified as eutrophic, overweight and obese, according to the curves of body mass index for age and sex proposed by the World Health Organization. The statistical analysis used was one-way ANOVA, followed by Scheffé's post-hoc test, with $p<0.01$.

Results: In boys, the largest increase occurred in the overweight group, followed by the obese and eutrophic groups. In girls, the largest increases occurred in the groups with overweight and eutrophic children, followed by the obese group.

Conclusion: During the 20-year period analyzed, there were changes in adiposity, even when the nutritional status was controlled, showing that individuals who have similar body mass indexes may vary in proportion and distribution of subcutaneous adipose tissue. In both sexes, the increase was higher in the overweight group, and mainly in central skinfolds.
\end{abstract}

J Pediatr (Rio J). 2012;88(3):239-45: Skinfolds thickness, adipose tissue, school-age children.

1. Mestrando(a), Centro de Estudos do Laboratório de Aptidão Física de São Caetano do Sul (CELAFISCS), São Caetano do Sul, SP

2. CELAFISCS, São Caetano do Sul, SP.

3. Mestre. CELAFISCS, São Caetano do Sul, SP.

4. Livre docente. CELAFISCS, São Caetano do Sul, SP.

Não foram declarados conflitos de interesse associados à publicação deste artigo.

Apoio financeiro: Fundação de Amparo à Pesquisa do Estado de São Paulo (FAPESP), sob o processo número 2010/20749-8.

Como citar este artigo: Ferrari TK, Ferrari GL, da Silva Jr JP, da Silva LJ, Oliveira LC, Matsudo VK. Modifications of adiposity in school-age children according to nutritional status: a 20-year analysis. J Pediatr (Rio J). 2012;88(3):239-45.

Artigo submetido em 21.12.11, aceito em 09.03.12.

http://dx.doi.org/10.2223/JPED.2191 


\section{Introdução}

Em alguns países, mudanças demográficas, socioeconômicas e epidemiológicas levaram a alterações nos padrões nutricionais, aumentando significativamente a prevalência de excesso de peso e obesidade, fenômeno este descrito como transição nutricional1,2. Diversos fatores, como as mudanças no estilo de vida, a alimentação e o aumento do estresse, associados ao avanço tecnológico, econômico e social ao longo das últimas décadas, acarretaram o aumento da obesidade em todo o mundo, constituindo um problema de saúde pública ${ }^{3}$. Em 2009, uma em cada três crianças de 5 a 9 anos de idade estava acima do peso. O excesso de peso foi observado em $33,5 \%$ das crianças brasileiras, sendo que $16,6 \%$ dos meninos e $11,8 \%$ das meninas eram obesas. Esses números representam um salto na prevalência de excesso de peso em crianças ao longo de 34 anos, sendo $10,9 \%$ em 1974-1975, 15\% em 1989 e 34,8\% em 20082009 nos meninos e 8,6\% em 1975-1975, 11,9\% em 1989 e $32 \%$ em 2008-2009 nas meninas ${ }^{4}$.

O desenvolvimento precoce de doenças crônicas não transmissíveis, como as cardiovasculares, hipertensão, elevados níveis de lipoproteínas de baixa densidade, entre outras, está associado com elevados níveis de adiposidade 5 . Algumas evidências apontam que o período de maior risco para incidência da obesidade é a transição entre a adolescência e as etapas precoces da vida adulta, em ambos os sexos e em vários grupos étnicos ${ }^{6}$.

Alguns estudos nacionais e internacionais vêm apresentando resultados das alterações da adiposidade ao longo dos anos, uma vez que esta tem sofrido modificações ${ }^{7-9}$. Estudos sugerem que a variação da distribuição anatômica da gordura corporal é um importante indicador morfológico, relacionado com complicações endócrinas e metabólicas predisponentes ao aparecimento e desenvolvimento de doenças cardiovasculares ${ }^{10}$. Indivíduos com disposição centrípeta da gordura corporal tendem a apresentar maior incidência de diabetes ${ }^{11,12}$, hipertensão ${ }^{13}$, síndrome metabólica e alterações desfavoráveis no perfil das lipoproteínas plasmáticas ${ }^{14-16}$. Apesar de a obesidade central ser mais fortemente associada com uma gama de morbidades, são poucos os estudos que buscam evidenciar essa distribuição da gordura corporal. Com isso, o objetivo do presente estudo foi analisar as modificações da adiposidade de escolares durante 20 anos, de acordo com o estado nutricional.

\section{Métodos}

O presente estudo faz parte do Projeto Misto Longitudinal de Crescimento, Desenvolvimento e Aptidão Física de Ilhabela, desenvolvido pelo Centro de Estudos do Laboratório de Aptidão Física de São Caetano do Sul (CELAFISCS) desde 1978, com o objetivo de estudar e acompanhar o crescimento, desenvolvimento e a aptidão física de escolares do município de Ilhabela (SP), Brasil, que possui uma população de 28.176 habitantes e uma área territorial de $348 \mathrm{~km}^{2} 17$. Este é o único projeto longitudinal realizado em países em desenvolvimento, cuja principal característica reside no uso de materiais não sofisticados, técnicas não complexas e a facilidade e simplicidade do método que permite a alta aplicabilidade em grandes grupos ${ }^{18}$. O projeto realiza uma avaliação semestral, sempre nos meses de abril e outubro, totalizando 65 avaliações e 18.000 registros até o presente momento, que incluem medidas antropométricas, testes neuromotores e metabólicos, nutricionais, posturais e do nível de atividade física.

Para compor a amostra deste estudo, foi analisado um banco de dados composto por mais de 16.000 escolares de ambos os sexos, com idade de 7 a 18 anos e que participaram das avaliações entre 1978 e 2011 , sendo que 1.094 escolares de ambos os sexos, de 7 a 10 anos, atendiam aos seguintes critérios de inclusão: (a) pelo menos uma avaliação completa em um dos períodos analisados; (b) estar no estágio pré-púbere de maturação sexual; (c) ser aparentemente saudável.

O projeto foi aprovado pelo Comitê de Ética da Universidade Federal de São Paulo (UNIFESP) sob o protocolo 0056/10, e todos os indivíduos tiveram o termo de consentimento livre e esclarecido assinado pelos pais ou responsáveis. Os escolares participaram das avaliações em um dos períodos analisados, e os dados selecionados fazem parte de um banco de dados de 1990/1991 (inicial), 2000/2001 (10 anos) e 2010/2011 (20 anos).

As variáveis analisadas foram: massa corporal ( $\mathrm{kg})$, estatura $(\mathrm{cm})$ e adiposidade $(\mathrm{mm})$ a partir da análise individual de cada dobra cutânea (DC), de acordo com a padronização CELAFISCS $^{19}$. A caracterização da amostra participante do estudo é apresentada na Tabela 1.

A medida da massa corporal $(\mathrm{kg})$ foi obtida mediante a utilização de uma balança digital com precisão de 100 gramas, com o indivíduo trajando o mínimo de roupas possível. A estatura $(\mathrm{cm})$ foi medida utilizando um estadiômetro e calculada pela média de três mensurações. Para o cálculo do índice de massa corporal (IMC), foram utilizadas as duas medidas anteriormente citadas, classificando os indivíduos em eutróficos, com excesso de peso e obesos, mediante as curvas propostas pela Organização Mundial da Saúde (OMS) ${ }^{20}$ de IMC para idade e sexo. Essa classificação tem sido utilizada para identificar o risco ou a condição efetiva de obesidade em populações ${ }^{1,8,13}$. Contudo, existem outros critérios para identificação de alterações do estado nutricional relacionados à adiposidade. Um dos métodos utilizados para esse fim é a medida das DCs, uma vez que a mesma tem apresentado alterações significativas no padrão de adiposidade9,18. O presente estudo buscou encontrar concordâncias ou divergências na utilização desses diferentes métodos em uma mesma população, uma vez que o método pode estar dando interpretações errôneas no que se refere à classificação, não demonstrando efetivamente alterações do fenômeno.

A adiposidade $(\mathrm{mm})$ foi obtida através da determinação de sete DCs (bíceps, tríceps, subescapular, suprailíaca, axilar média, abdome e panturrilha), que foram realizadas pelo mesmo avaliador em todas as avaliações do projeto. As medidas foram realizadas no hemicorpo direito do avaliado, 
Tabela 1 - Caracterização da amostra de acordo com o sexo, independentemente do período analisado

\begin{tabular}{lccccc}
\hline Sexo & Idade (anos) & Massa corporal (kg) & Estatura (cm) & Eutrófico (n) & Excesso de peso (n) \\
\hline Masculino & $8,72 \pm 1,12$ & $30,32 \pm 7,71$ & $132,97 \pm 8,7$ & 361 \\
Feminino & $8,58 \pm 1,08$ & $30,94 \pm 8,22$ & $133,15 \pm 9,27$ & 428 \\
\hline
\end{tabular}

sendo que foram realizadas três medidas sucessivas no mesmo local, considerando-se a média das três como o valor adotado. O modelo de compasso utilizado foi o Harpenden ${ }^{\circledR}$ calibrado previamente.

Os valores de reprodutibilidade e objetividade de cada medida realizada entre os anos variaram de 0,96 a 0,99 na massa corporal, 0,97 a 0,99 na estatura e 0,81 a 0,99 nas DCs.

Para a determinação do estágio de maturação biológica, foi utilizado o método de Tanner ${ }^{21}$ mediante a técnica de autoavaliação das características sexuais secundárias de acordo com o sexo, já validado $(0,60$ a 0,71$)$ no próprio Projeto de Ilhabela22.

Os dados foram apresentados como média $(\bar{X})$ e desvio padrão. Para verificar a normalidade dos dados, foi realizada a análise Kolmogorov-Smirnov. Para os dados paramétricos, foi realizada a Anova One-Way seguida do post-hoc de Scheffé. Para verificar a magnitude da diferença entre os períodos inicial e 20 anos, foi calculado o delta percentual $(\Delta \%)$. 0 nível de significância adotado foi de $p<0,01$. O software utilizado foi o Statistical Package for the Social Sciences (SPSS) versão 18.0 .

\section{Resultados}

A Tabela 2 mostra que houve um aumento em todas as variáveis analisadas, exceto nas DCs de tríceps e panturrilha no sexo masculino. No sexo masculino, estatura e DC axilar média foram significativamente $(p<0,01)$ diferentes durante o período de 20 anos e sofreram um aumento linear com o passar dos anos. Os maiores aumentos no sexo masculino aconteceram nas DCs axilar média e abdome.

Já no sexo feminino, a massa corporal foi significativamente $(p<0,01)$ diferente no período entre 10 e 20 anos. DC de tríceps e panturrilha foram significativamente $(p<0,01)$ diferentes entre o período inicial e 10 anos e diminuíram com o passar dos anos. DC subescapular, suprailíaca, axilar média, abdome e X $7 D C$ (adiposidade média das sete DCs estudadas) foram significativamente $(p<0,01)$ diferentes e aumentaram no período de 20 anos. DC de tríceps, suprailíaca, axilar média, abdome, panturrilha e $\bar{X} 7 D C$ foram significativamente $(p<0,01)$ diferentes durante o período entre 10 e 20 anos. Os maiores aumentos aconteceram nas DC axilar média (26,6\%), suprailíaca $(32,9 \%)$ e abdome $(51,2 \%)$.
A Tabela 3 mostra que houve um aumento em todas as variáveis analisadas em ambos os sexos, exceto na DC panturrilha no sexo feminino. Estatura, massa corporal, DC de bíceps, subescapular, suprailíaca, axilar média, abdome, panturrilha e $\bar{X} 7 D C$ foram significativamente $(p<0,01)$ diferentes durante o período de 20 anos no sexo masculino. Os maiores aumentos aconteceram nas DC suprailíaca (83\%), axilar média $(96,8 \%)$ e abdome $(105,4 \%)$.

Já no sexo feminino, a DC suprailíaca, abdome e $\bar{X} 7 D C$ foram significativamente $(p<0,01)$ diferentes entre o período inicial e 10 anos. DC suprailíaca, abdome e $\bar{X} 7 D C$ foram significativamente $(p<0,01)$ diferentes entre o período inicial e 20 anos. Os maiores aumentos no sexo feminino aconteceram nas DC suprailíaca $(33,1 \%)$, axilar média $(37 \%)$ e abdome $(48,6 \%)$.

A Tabela 4 mostra que houve um aumento em todas as variáveis analisadas no sexo masculino e em todas, exceto massa corporal, estatura e DC subescapular, no sexo feminino. DC de bíceps, subescapular, abdome e $\bar{X} 7 D C$ foram significativamente $(p<0,01)$ diferentes no sexo masculino durante o período de 20 anos. Os maiores aumentos no sexo masculino aconteceram nas DC axilar média $(49,2 \%)$, abdome $(51 \%)$ e subescapular $(53,8 \%)$. Apesar de não terem sido encontradas diferenças estatísticas em valores absolutos no sexo feminino, em valores percentuais houve grandes aumentos nas DC axilar média $(15,4 \%)$, suprailíaca $(22,8 \%)$ e abdome $(23,6 \%)$.

Para comparar as alterações da adiposidade de cada grupo, foi feita a média dos $\Delta \%$ de todas as DC. No sexo masculino, o maior aumento ocorreu no grupo com excesso de peso $(65,6 \%)$, seguido pelos grupos obesos $(39,8 \%)$ e eutróficos $(6,31 \%)$. Já no sexo feminino, os maiores aumentos ocorreram de forma similar nos grupos com excesso de peso $(20,9 \%)$ e eutróficos $(19,6 \%)$, seguidos pelo grupo obesos (11\%).

\section{Discussão}

Os resultados do presente estudo mostraram que o acúmulo de gordura subcutânea central tem aumentado de forma mais acentuada do que a adiposidade total avaliada a partir do IMC, ou seja, mesmo em populações em que não houve alteração no IMC, podem ter ocorrido mudanças desfavoráveis na composição corporal e distribuição da gordura corporal para um perfil associado com maiores riscos de doenças. No sexo 
Tabela 2 - Análise descritiva e comparativa das variáveis antropométricas de escolares de ambos os sexos, classificados como eutróficos de acordo com o período de avaliação (inicial, 10 e 20 anos)

\begin{tabular}{|c|c|c|c|c|}
\hline & Inicial & 10 anos & 20 anos & $\Delta \%$ \\
\hline \multicolumn{5}{|l|}{ Masculino } \\
\hline Massa corporal (kg) & $27,01 \pm 3,84$ & $26,64 \pm 4,28$ & $27,67 \pm 4,42$ & 2,44 \\
\hline Estatura (cm) & $130,01 \pm 7,61$ & $132,29 \pm 7,45$ & $133,13 \pm 8,48^{+}$ & 2,39 \\
\hline Bíceps (mm) & $4,75 \pm 1,15$ & $4,87 \pm 1,46$ & $5,32 \pm 2,04$ & 12,00 \\
\hline Tríceps (mm) & $9,12 \pm 2,16$ & $8,05 \pm 2,57$ & $8,46 \pm 2,63$ & $-7,23$ \\
\hline Subescapular (mm) & $5,39 \pm 1,11$ & $5,59 \pm 1,32$ & $5,79 \pm 1,97$ & 7,42 \\
\hline Suprailíaca (mm) & $4,87 \pm 1,35$ & $4,86 \pm 1,52$ & $5,33 \pm 2,04$ & 9,44 \\
\hline Axilar média (mm) & $4,27 \pm 0,92$ & $4,55 \pm 1,21$ & $4,81 \pm 1,81^{\dagger}$ & 12,64 \\
\hline Abdome (mm) & $6,36 \pm 2,13$ & $6,24 \pm 2,88$ & $7,33 \pm 3,21$ & 15,25 \\
\hline Panturrilha (mm) & $9,23 \pm 2,77$ & $7,92 \pm 2,46$ & $8,74 \pm 2,98$ & $-5,30$ \\
\hline $\bar{X} 7 D C(\mathrm{~mm})$ & $6,28 \pm 1,35$ & $6,01 \pm 1,71$ & $6,54 \pm 2,03$ & 4,14 \\
\hline \multicolumn{5}{|l|}{ Feminino } \\
\hline Massa corporal (kg) & $27,52 \pm 4,71$ & $25,90 \pm 4,68$ & $28,55 \pm 5,91^{\ddagger}$ & 3,74 \\
\hline Estatura (cm) & $130,58 \pm 8,22$ & $130,63 \pm 7,62$ & $133,21 \pm 9,92$ & 2,01 \\
\hline Bíceps (mm) & $5,94 \pm 1,67$ & $5,98 \pm 1,74$ & $6,62 \pm 2,49$ & 11,44 \\
\hline Tríceps (mm) & $11,09 \pm 2,46$ & $9,77 \pm 2,38^{*}$ & $11,23 \pm 3,26^{\ddagger}$ & 1,26 \\
\hline Subescapular (mm) & $6,27 \pm 1,67$ & $6,54 \pm 1,68$ & $7,12 \pm 2,72^{+}$ & 13,55 \\
\hline Suprailíaca (mm) & $5,92 \pm 2,33$ & $6,33 \pm 2,31$ & $7,87 \pm 3,49^{\dagger \neq}$ & 32,93 \\
\hline Axilar média (mm) & $5,08 \pm 1,50$ & $5,41 \pm 1,58$ & $6,43 \pm 2,78^{\dagger \neq}$ & 26,57 \\
\hline Abdome (mm) & $7,63 \pm 2,98$ & $8,38 \pm 3,81$ & $11,54 \pm 5,15^{\dagger \neq}$ & 51,24 \\
\hline Panturrilha (mm) & $11,65 \pm 3,25$ & $10,04 \pm 2,93^{*}$ & $11,75 \pm 3,73^{\ddagger}$ & 0,85 \\
\hline $\bar{X} 7 D C(\mathrm{~mm})$ & $7,65 \pm 1,87$ & $7,49 \pm 2,01$ & $8,93 \pm 2,88^{\dagger \neq}$ & 16,73 \\
\hline
\end{tabular}

$\Delta \%=$ delta percentual.

$\overline{\mathrm{X}} 7 \mathrm{DC}=$ adiposidade média das sete DCs (bíceps, tríceps, subescapular, suprailíaca, axilar média, abdome e panturrilha).

$\mathrm{p}<0,01$.

* Inicial diferente de 10 anos.

$\dagger$ Inicial diferente de 20 anos.

¥ 10 anos diferente de 20 anos.

Tabela 3 - Análise descritiva e comparativa das variáveis antropométricas de escolares de ambos os sexos, classificados como excesso de peso de acordo com o período de avaliação (inicial, 10 e 20 anos)

\begin{tabular}{|c|c|c|c|c|}
\hline & Inicial & 10 anos & 20 anos & $\Delta \%$ \\
\hline \multicolumn{5}{|l|}{ Masculino } \\
\hline Massa corporal $(\mathrm{kg})$ & $32,02 \pm 5,62$ & $33,48 \pm 7,34$ & $37,14 \pm 6,43^{+}$ & 15,99 \\
\hline Estatura (cm) & $130,70 \pm 9,35$ & $133,52 \pm 10,67$ & $138,45 \pm 9,78^{+}$ & 5,92 \\
\hline Bíceps (mm) & $6,06 \pm 1,55$ & $8,54 \pm 9,37$ & $9,37 \pm 3,04^{+}$ & 54,62 \\
\hline Tríceps (mm) & $11,94 \pm 2,46$ & $13,24 \pm 3,75$ & $14,54 \pm 5,45$ & 21,77 \\
\hline Subescapular (mm) & $6,22 \pm 1,10$ & $7,62 \pm 2,17$ & $9,90 \pm 4,47^{+}$ & 59,16 \\
\hline Suprailíaca (mm) & $6,30 \pm 2,27$ & $9,56 \pm 5,75$ & $11,53 \pm 5,62^{\dagger}$ & 83,01 \\
\hline Axilar média $(\mathrm{mm})$ & $5,08 \pm 1,52$ & $6,88 \pm 3,54$ & $10,00 \pm 4,50^{\dagger}$ & 96,85 \\
\hline Abdome (mm) & $8,81 \pm 4,16$ & $14,38 \pm 8,12$ & $18,10 \pm 7,39^{+}$ & 105,44 \\
\hline Panturrilha (mm) & $11,92 \pm 2,89$ & $14,58 \pm 4,84$ & $16,51 \pm 6,51^{\dagger}$ & 38,50 \\
\hline $\bar{X} 7 \mathrm{DC}(\mathrm{mm})$ & $8,05 \pm 2,03$ & $10,68 \pm 4,56$ & $12,83 \pm 4,25^{\dagger}$ & 59,37 \\
\hline \multicolumn{5}{|l|}{ Feminino } \\
\hline Massa corporal $(\mathrm{kg})$ & $35,07 \pm 5,73$ & $35,19 \pm 5,90$ & $37,48 \pm 7,70$ & 6,87 \\
\hline Estatura $(\mathrm{cm})$ & $133,89 \pm 8,35$ & $135,07 \pm 9,13$ & $137,79 \pm 10,97$ & 2,91 \\
\hline Bíceps (mm) & $9,24 \pm 2,79$ & $11,12 \pm 2,81$ & $9,93 \pm 2,99$ & 7,46 \\
\hline Tríceps (mm) & $15,98 \pm 3,97$ & $17,13 \pm 3,21$ & $16,48 \pm 3,02$ & 3,12 \\
\hline Subescapular (mm) & $10,74 \pm 3,87$ & $12,76 \pm 3,44$ & $12,75 \pm 4,61$ & 18,71 \\
\hline Suprailíaca (mm) & $11,81 \pm 4,72$ & $16,53 \pm 4,40^{*}$ & $15,73 \pm 5,81^{\dagger}$ & 33,19 \\
\hline Axilar média $(\mathrm{mm})$ & $9,06 \pm 4,47$ & $11,81 \pm 3,14$ & $12,42 \pm 5,08^{\dagger}$ & 37,08 \\
\hline Abdome (mm) & $15,00 \pm 6,24$ & $21,34 \pm 4,98^{*}$ & $22,30 \pm 5,81^{\dagger}$ & 48,66 \\
\hline Panturrilha $(\mathrm{mm})$ & $17,24 \pm 3,83$ & $17,43 \pm 3,96$ & $17,01 \pm 5,42$ & $-1,33$ \\
\hline $\bar{X} 7 D C(\mathrm{~mm})$ & $12,72 \pm 3,66$ & $15,44 \pm 2,87$ & $15,23 \pm 3,63^{+}$ & 19,73 \\
\hline
\end{tabular}

$\Delta \%=$ delta percentual.

$\overline{\mathrm{X}} 7 \mathrm{DC}=$ adiposidade média das sete DCs (bíceps, tríceps, subescapular, suprailíaca, axilar média, abdome e panturrilha).

$\mathrm{p}<0,01$.

* Inicial diferente de 10 anos.

$\dagger$ Inicial diferente de 20 anos.

₹ 10 anos diferente de 20 anos. 
Tabela 4 - Análise descritiva e comparativa das variáveis antropométricas de escolares de ambos os sexos, classificados como obesos de acordo com o período de avaliação (inicial, 10 e 20 anos)

\begin{tabular}{|c|c|c|c|c|}
\hline & Inicial & 10 anos & 20 anos & $\Delta \%$ \\
\hline \multicolumn{5}{|l|}{ Masculino } \\
\hline Massa corporal $(\mathrm{kg})$ & $40,07 \pm 7,67$ & $45,33 \pm 9,09$ & $45,08 \pm 8,22$ & 12,50 \\
\hline Estatura $(\mathrm{cm})$ & $135,58 \pm 9,59$ & $138,59 \pm 7,57$ & $137,91 \pm 8,61$ & 1,71 \\
\hline Bíceps (mm) & $9,82 \pm 3,66$ & $11,51 \pm 3,76$ & $14,32 \pm 4,01^{\dagger}$ & 45,82 \\
\hline Tríceps (mm) & $18,04 \pm 5,91$ & $18,72 \pm 4,23$ & $22,57 \pm 5,63$ & 25,11 \\
\hline Subescapular (mm) & $12,08 \pm 5,44$ & $17,96 \pm 7,41$ & $18,59 \pm 6,98^{\dagger}$ & 53,89 \\
\hline Suprailíaca (mm) & $16,56 \pm 9,57$ & $20,37 \pm 9,48$ & $20,81 \pm 7,95$ & 25,66 \\
\hline Axilar média (mm) & $10,56 \pm 4,75$ & $17,02 \pm 9,73$ & $15,76 \pm 5,12$ & 49,24 \\
\hline Abdome $(\mathrm{mm})$ & $20,31 \pm 9,44$ & $27,78 \pm 13,81$ & $30,67 \pm 7,30^{+}$ & 51,00 \\
\hline Panturrilha (mm) & $16,66 \pm 7,35$ & $22,60 \pm 7,50$ & $21,34 \pm 4,51$ & 28,09 \\
\hline$\overline{\mathrm{X}} 7 \mathrm{DC}(\mathrm{mm})$ & $14,86 \pm 5,65$ & $19,42 \pm 7,05$ & $20,51 \pm 4,74^{+}$ & 38,02 \\
\hline \multicolumn{5}{|l|}{ Feminino } \\
\hline Massa corporal $(\mathrm{kg})$ & $46,42 \pm 7,41$ & $43,51 \pm 6,65$ & $44,77 \pm 7,87$ & $-3,55$ \\
\hline Estatura $(\mathrm{cm})$ & $140,51 \pm 8,10$ & $137,41 \pm 6,77$ & $137,97 \pm 8,59$ & $-1,80$ \\
\hline Bíceps (mm) & $13,15 \pm 2,28$ & $15,37 \pm 3,80$ & $13,94 \pm 3,88$ & 6,00 \\
\hline Tríceps (mm) & $21,59 \pm 2,76$ & $24,02 \pm 3,71$ & $21,97 \pm 5,39$ & 1,76 \\
\hline Subescapular (mm) & $20,52 \pm 5,68$ & $20,75 \pm 5,36$ & $19,07 \pm 6,42$ & $-7,06$ \\
\hline Suprailíaca (mm) & $21,24 \pm 4,77$ & $27,28 \pm 4,85$ & $26,10 \pm 7,06$ & 22,88 \\
\hline Axilar média (mm) & $15,88 \pm 3,81$ & $21,85 \pm 6,22$ & $18,33 \pm 5,56$ & 15,42 \\
\hline Abdome $(\mathrm{mm})$ & $26,69 \pm 3,87$ & $31,33 \pm 6,32$ & $33,01 \pm 7,98$ & 23,67 \\
\hline Panturrilha (mm) & $20,53 \pm 10,13$ & $23,51 \pm 5,30$ & $23,54 \pm 6,79$ & 14,66 \\
\hline $\bar{X} 7 \mathrm{DC}(\mathrm{mm})$ & $19,94 \pm 3,38$ & $23,44 \pm 4,43$ & $22,25 \pm 4,98$ & 11,58 \\
\hline
\end{tabular}

$\Delta \%=$ delta percentual.

$\overline{\mathrm{X}} 7 \mathrm{DC}=$ adiposidade média das sete DCs (bíceps, tríceps, subescapular, suprailíaca, axilar média, abdome e panturrilha).

$\mathrm{p}<0,01$.

* Inicial diferente de 10 anos.

$\dagger$ Inicial diferente de 20 anos.

¥ 10 anos diferente de 20 anos.

masculino, o grupo com excesso de peso teve maior aumento de DC do que o grupo obesos, destacando um aumento de DC inclusive no grupo classificado como eutróficos. Já no sexo feminino, esses resultados foram ainda mais evidentes, pois demonstraram maior aumento de DC no grupo eutróficas e com excesso de peso do que no grupo obesas, indicando que apenas o critério de classificação de estado nutricional pode não refletir claramente as modificações do acúmulo do tecido adiposo ou aumento da obesidade.

Um estudo australiano ${ }^{23}$ que investigou a tendência secular (1985-1997-2002) das DC abdominal e de tríceps em crianças de 10 a 12 anos mostrou que, no período de 20 anos, houve um aumento de $12 \%$ nos meninos e $27 \%$ nas meninas na DC abdominal e de $3 \%$ nos meninos e $8 \%$ nas meninas na DC de tríceps. O presente estudo apresentou um aumento maior na DC abdominal no período de 20 anos analisado, sendo 57,2\% nos meninos e $49 \%$ nas meninas. Já a DC de tríceps apresentou um aumento de $6,5 \%$ nos meninos e de $8,4 \%$ nas meninas. Assim como no presente estudo, em que os maiores aumentos ocorreram nas DC centrais, os dados demonstrados sugerem uma mudança na forma do corpo, independente de um aumento do IMC e uma mudança em direção a um perfil associado com maior risco de doenças ${ }^{24}$.
Garnett ${ }^{25}$ analisou a prevalência do aumento da obesidade central, a partir da circunferência da cintura e da relação altura/ cintura, em escolares de 7 a 15 anos, no período de 1985 e 2007, e também mostrou que, em 20 anos, a adiposidade central aumentou em um ritmo mais rápido que a adiposidade total, sendo que esse aumento foi maior no sexo feminino. Apesar de os autores utilizarem a circunferência abdominal e a relação altura/cintura, esses dados confirmam os obtidos no presente estudo, embora tenha sido encontrado um maior aumento principalmente no sexo masculino.

Em países desenvolvidos, Olds ${ }^{9}$ analisou a tendência secular da adiposidade e de sua distribuição em crianças e adolescentes de 0 a 18 anos. Os resultados apresentaram um aumento na espessura das DC de tríceps e subescapular no período de 1951 a 2003, sendo que ocorreu uma diminuição na relação tricipital/subescapular (T/S), o que representa uma distribuição mais centralizada da gordura. Quando comparados os sexos, essa diminuição foi maior nas meninas do que nos meninos. Esses dados apoiam os encontrados no presente estudo, uma vez que a DC subescapular teve um aumento maior que a DC de tríceps em meninos eutróficos ( $\mathrm{S}=7,4 \%$; $\mathrm{T}=-7,2 \%$ ), com excesso de peso ( $\mathrm{S}=59,1 \%$; $\mathrm{T}=21,7 \%$ ) e entre os obesos ( $\mathrm{S}=53,8 \% ; \mathrm{T}=25,1 \%$ ), assim como em meninas eutróficas ( $\mathrm{S}=13,5 \%$; $\mathrm{T}=1,2 \%$ ) 
e com excesso de peso ( $\mathrm{S}=18,7 \% ; \mathrm{T}=3,1 \%$ ), durante o período de 20 anos analisado. Porém, o presente estudo apresenta um maior aumento da adiposidade central nos meninos em relação às meninas.

Analisando o comportamento da espessura das DC de tríceps e subescapular de escolares de 7 a 11 anos, nos anos de 1972 e 1973, Hegg 26 mostrou que a DC de tríceps apresentou valores mais elevados do que a DC subescapular em ambos os sexos. Esses dados diferem dos encontrados no presente estudo, uma vez que a DC subescapular teve um aumento mais elevado do que a DC de tríceps nos três períodos analisados (inicial, 10 e 20 anos) em ambos os sexos. Esses resultados demonstram uma possível alteração da distribuição do tecido adiposo subcutâneo no decorrer do tempo.

Janssen ${ }^{27}$ estimou a prevalência da obesidade abdominal a partir da circunferência da cintura em adolescentes e adultos no período entre 1981 e 2009, mostrando que a obesidade abdominal aumentou com a idade e foi maior em mulheres. $O$ autor também estimou a prevalência da obesidade abdominal em adultos e adolescentes classificados a partir do IMC como normais, com excesso de peso e obesos. A prevalência de obesidade abdominal foi de $2,6 \%$ nos eutróficos, $35,3 \%$ nos com excesso de peso e $93 \%$ entre os obesos. Apesar de analisar uma idade superior, esses dados foram semelhantes aos encontrados no presente estudo, uma vez que a gordura subcutânea localizada na região central teve a prevalência aumentada durante o período analisado.

Apesar de o presente estudo apresentar uma análise de 20 anos em um projeto misto longitudinal realizado em país em desenvolvimento há mais de 30 anos e possuir uma amostra considerável de 1.915 crianças, os autores consideram que a investigação atual possui algumas limitações: a) a abrangência da amostra, uma vez que a mesma é limitada geograficamente; b) falta de critérios para classificar o IMC de crianças brasileiras por idade e sexo; c) avaliação indireta da adiposidade, mesmo sendo realizada pelo mesmo avaliador em todos os períodos analisados; d) o não controle do nível socioeconômico, apesar de a obesidade não ser explicada pelo crescimento econômico de uma região ${ }^{28}$; e) falta de um consenso para embasar a discussão sobre o critério metodológico para avaliar a adiposidade em crianças; e f) não ter sido realizada avaliação da massa magra.

Após uma análise de 20 anos, os autores concluíram que o tecido adiposo teve aumento significativo em ambos os sexos e estados nutricionais. Houve modificações da adiposidade, mesmo com o controle do estado nutricional, durante o período de 20 anos analisado, mostrando que os indivíduos podem ter IMC semelhantes, mas ao mesmo tempo variar em proporção e distribuição de tecido adiposo subcutâneo. No sexo masculino, o maior aumento de DC ocorreu no grupo com excesso de peso, seguido pelos grupos obesos e eutróficos após um período de 20 anos. Já no sexo feminino, os maiores aumentos ocorreram de forma similar nos grupos com excesso de peso e eutróficas, seguidos pelo grupo obesas. Em ambos os sexos, o aumento foi maior nas DC centrais, quando comparadas com as DC periféricas. Assim, é sugerida uma possível alteração da composição corporal, em que pode estar ocorrendo um aumento na massa gorda.

\section{Agradecimentos}

À apoiadora do projeto, Fundação de Amparo à Pesquisa do Estado de São Paulo, sob o processo número 2010/20749-8; aos participantes, pais e responsáveis; aos professores e à coordenação da Escola Municipal José Antônio Verzegnassi e da Escola Municipal Eva Esperança; e à direção da Prefeitura Municipal de Ilhabela.

\section{Referências}

1. de Franca E, Alves JG. Dyslipidemia among adolescents and children from Pernambuco. Arq Bras Cardiol. 2006;87:722-7.

2. Brandão AP, Brandão AA, Berenson GS, Fuster V. Metabolic syndrome in children and adolescents. Arq Bras Cardiol. 2005; 85:79-81.

3. Department of Health Public Health Research Consortium, Law C, Power C, Graham H, Merrick D. Obesity and health inequalities. Obes Rev. 2007;8:19-22.

4. Instituto Brasileiro de Geografia e Estatística (IBGE). Pesquisa de Orçamentos Familiares 2008-2009: antropometria e estado nutricional de crianças, adolescentes e adultos no Brasil. Rio de Janeiro: IBGE; 2010.

5. Campbell I. The obesity epidemic: can we turn the tide? Heart. 2003;89:ii22-4.

6. Gordon-Larsen P, Adair LS, Nelson MC, Popkin BM. Five-year obesity incidence in the transition period between adolescence and adulthood: the National Longitudinal Study of Adolescent Health. Am J Clin Nutr. 2004;80:569-75.

7. Pinto MC, Oliveira AC. Occurrence of child obesity in preschool children in a São Paulo day-care center. Einstein (São Paulo). 2009; 7:170-5.

8. Moraes SA, Beltrán Rosas J, Mondini L, Freitas IC. Prevalence of overweight and obesity, and associated factors in school children from urban area in Chilpancingo, Guerrero, Mexico, 2004. Cad Saude Publica. 2006;22:1289-301.

9. Olds TS. One million skinfolds: secular trends in the fatness of young people 1951-2004. Eur J Clin Nutr. 2009;63:934-46.

10. Canoy D. Distribution of body fat and risk of coronary heart disease in men and women. Curr Opin Cardiol. 2008;23:591-8.

11. Vazquez G, Duval S, Jacobs DR Jr, Silventoinen K. Comparison of body mass index, waist circumference, and waist/hip ratio in predicting incident diabetes: a meta-analysis. Epidemiol Rev. 2007;29:115-28.

12. Ehtisham S, Crabtree N, Clark P, Shaw N, Barrett T. Ethnic differences in insulin resistance and body composition in United Kingdom adolescents. J Clin Endocrinol Metab. 2005;90:3963-9.

13. Gus M, Fuchs SC, Moreira LB, Moraes RS, Wiehe M, Silva AF, et al. Association between different measurements of obesity and the incidence of hypertension. Am J Hypertens. 2004;17:50-3.

14. Souza MS, Leme RB, Franco RR, Romaldini CC, Tumas R, Cardoso $A L$, et al. Síndrome metabólica em adolescentes com sobrepeso e obesidade. Rev Paul Pediatr. 2007;25:214-20.

15. Jung C, Fischer N, Fritzenwanger M, Figulla HR. Anthropometric indices as predictors of the metabolic syndrome and its components in adolescents. Pediatr Int. 2010;52:402-9.

16. Oliveira CL, Mello MT, Cintra IP, Fisberg M. Obesidade e síndrome metabólica na infância e adolescência. Rev Nutr. 2004; 17:237-45. 
17. Instituto Brasileiro de Geografia e Estatística (IBGE). Censo Demográfico 2007: Resultados do universo. http://www.ibge. gov.br. Acesso: 20/04/11.

18. Ferrari GL, Hespanhol JE, Arruda M. Associação dos indicadores da composição corporal com a maturação sexual em jovens atletas futebolistas. Rev Bras Ciênc Saúde. 2007;3:3-7.

19. Matsudo VK. Testes em Ciências do Esporte. 7a ed. São Caetano do Sul: Centro de Estudos do Laboratório de Aptidão Física de São Caetano do Sul; 2005.

20. de Onis M, Onyango AW, Borghi E, Siyam A, Nishida C, Siekmann J. Development of a WHO growth reference for school-aged children and adolescents. Bull World Health Organ. 2007;85:660-7.

21. Tanner JM. Growth at adolescence. Oxford: Blackwell Scientific. In: Malina RM, Bouchard C. Growth, maturation, and physical activity. Champaign: Human Kinetics Books; 1991.

22. Matsudo SM, Matsudo VK. Self-assessment and physician assessment of sexual maturation in Brazilian boys and girls: concordance and reproducibility. Am J Hum Biol. 1994;6:451-5.

23. Dollman J, Olds TS. Secular changes in fatness and fat distribution in Australian children matched for body size. Int J Pediatr Obes. 2006;1:109-13.

24. Lobstein T, Baur L, Uauy R; IASO International Obesity TaskForce. Obesity in children and young people: a crisis in public health. Obes Rev. 2004;5:4-104.
25. Garnett SP, Baur LA, Cowell CT. The prevalence of increased central adiposity in Australian school children 1985 to 2007. Obes Rev. 2011;12:887-96.

26. Hegg RV. Skinfolds in school children from 7 to 11 years of age. Rev Saude Publica. 1978;12:147-50.

27. Janssen I, Shields M, Craig CL, Tremblay MS. Prevalence and secular changes in abdominal obesity in Canadian adolescents and adults, 1981 to 2007-2009. Obes Rev. 2011;12:397-405.

28. Bua J, Olsen LW, Sørensen TI. Secular trends in childhood obesity in Denmark during 50 years in relation to economic growth. Obesity (Silver Spring). 2007;15:977-85.

Correspondência:

Tatiane K. Ferrari

Rua Heloísa Pamplona, 269, sala 31, Fundação

CEP 09520-320 - São Caetano do Sul, SP

Tel.: (11) 4229.8980, (11) 4229.9643

E-mail: celafiscs@celafiscs.org.br, tatianekferrari@yahoo.com.br 